\title{
Loading Algorithms for Adaptive SS-MC-MA Systems over Wireline Channels: Comparison with DMT*
}

\author{
Matthieu Crussière, Jean-Yves Baudais, Jean-François Hélard \\ Electronics and Telecommunications Institute of Rennes (IETR), CS 14215, 35043 Rennes Cedex, France. \\ matthieu.crussiere@insa-rennes.fr
}

\begin{abstract}
In this paper, we propose to combine adaptive loading principles with the spread-spectrum multicarrier multiple access (SS-MC-MA) scheme. Such an approach has particular interests in the context of powerline communications (PLC), where the transmitter has not only to exploit robust transmission techniques, but has also to adapt the waveform to the channel response. We introduce finite-granularity loading algorithms that dynamically handle the configuration of the system under power spectral density constraints. The presented algorithms assign subcarriers, spreading codes, bits and energy to each user in order to maximize either the data rate or the noise margin at a given target symbol error rate. These algorithms can actually be viewed as a widening of the classical waterfilling approach in the case of an hybrid spread-spectrum multicarrier system. Simulation results of the new scheme are presented for different measured PLC channels and are compared with those of the classical discrete multitone modulation (DMT) approach. It is shown that the adaptive SS-MC-MA scheme performs significantly better than DMT, due to its natural energy gathering capability. Adaptive SS-MC-MA then leads to a more efficient bits and energies distribution and constitutes a simple solution to reduce the quantification loss induced by the use of finite order modulations.
\end{abstract}

\section{INTRODUCTION}

In the past few years, the intense demand for highspeed home Internet has given rise to permanent necessity for additional transmission capacities on the access network. High data rate communications over the so-called "last-mile" have become a challenging task and have motivated the study of new telecommunication networks and new transmission technologies. A promising possibility is then offered by powerline communications (PLC), which are today considered as a convenient and cheap alternative to already available digital subscriber line (DSL), cable or wireless technologies.

However, power distribution networks present an unfavorable transmission medium, since they have not been designed for communication purposes. The power line channel exhibits multipaths caused by reflections on the discontinuities of the tree-like-structured network, and is characterized by strong frequency-dependent cable losses [2]. Unfavorable noise conditions have also to be considered among which the most unpopular impulsive noise [3]. By their remarkable robustness against frequency dispersive channels, multicarrier techniques, and in particular digital

* Parts of this paper were presented at the fi fth International Worshop on Multi-Carrier Spread Spectrum \& Related Topics (MCSS 2005), Septembre 2005, Oberpfaffenhofen, Germany [1]. multitone (DMT), are then largely proposed for communications over the power grid [4], [5]. But in the particular case of outdoor powerline communications, multicellular and multiuser needs have moreover to be answered, and multicarrier spread spectrum (MC-SS) schemes also represent suitable schemes to investigate, as proposed in this paper. These hybrid techniques have already shown very good performances in the case of multiuser communications in diffi cult environments [6] and can therefore be considered as potential solutions for powerline communications. Furthermore, the spread spectrum component of MC-SS has already been shown to present a good impulsive noise mitigation effect [7]. But beyond these performance considerations, we will show hereafter that MC-SS systems can, if judiciously dimensioned, offer additionnal interests in terms of resource allocation.

Contrary to wireless channels, wireline channels essentially offer quasi-static impulse responses, which implies that the channel state information (CSI) can be known at the transmitter side. Under this assumption, adaptive resource allocation procedures can be followed before the transmission, resulting in significative improvements in terms of system throughput or robustness [8]. Many subcarrier loading algorithms have been developed on the basis of the well-known optimal waterfilling solution for allocat- 
ing power and bits in DMT $[9,10]$. They are today namely exploited in DSL systems. The general principle basically consists in assigning an appropriate energy/constellation pair to each subcarrier according to the signal-to-noise ratio (SNR) achieved per subcarrier. However, for long lines or deep fades, the subcarrier SNR can drop under a certain threshold resulting in unload situations. Moreover, the use of fi nite order constellations like QAM, combined with power spectral density (PSD) limitations brings about a quantifi cation loss that implies a global achievable rate reduction. To circumvent this problem, fractionnal bits techniques exploiting trellis coded modulations with variable rates can be carried out, but lead to an important increase of complexity due to channel coding scheme modifi cations.

In this article, we will show that combining adaptive loading principles with MC-SS systems allows to minimize the quantifi cation loss effects at a reasonable complexity cost. More precisely, the particular MC-SS combination commonly referred to as spread-spectrum multicarrier multiple-access (SS-MC-MA) [11] will be demonstrated to present intrinsec abilities for effi cient resource allocation. Taking into account the spreading component of the SS-MC-MA waveform, new allocation algorithms will therefore be introduced to handle the system confi guration and maximize either the throughput or the noise margin of each user of the network. As it will be detailed, these algorithms translate into subcarriers, codes, bits and energies assignment procedures that turn out to be some generalization of the waterfi lling solution to the case when subcarriers are merged and bound with spreading sequences.

This paper is organized as follows. Section 2 describes the proposed adaptive SS-MC-MA system. The allocation problem formulation is then stated in section 3. Sections 4 and 5 introduce the allocation principles associated to both of the cases throughput and noise margin maximization tasks, respectively. An arbitrary subcarrier distribution among users is first considered in these sections and the subcarrier allocation algorithm is indeed presented in section 6 . The system performance is then analyzed in section 7 and compared with that obtained with the classical DMT system. Finally, section 8 concludes this paper.

\section{ADAPTIVE SYSTEM DESCRIPTION}

As mentioned in the introductive section, it is proposed to combine adaptive resource allocation principles to a particular version of MC-SS systems, called SS-MC-MA. Let us recall that SS-MC-MA is a multicarrier modulation that combines spread-spectrum and frequency division multiple access (FDMA). The FDMA component is based on the transmission of several subsets of subcarriers in parallel, each subset being exclusively assigned to a single user. The SS component allows each user to simultaneously transmit several symbols on the same subset by spreading them in the frequency domain. As each subcarrier is used by a sole

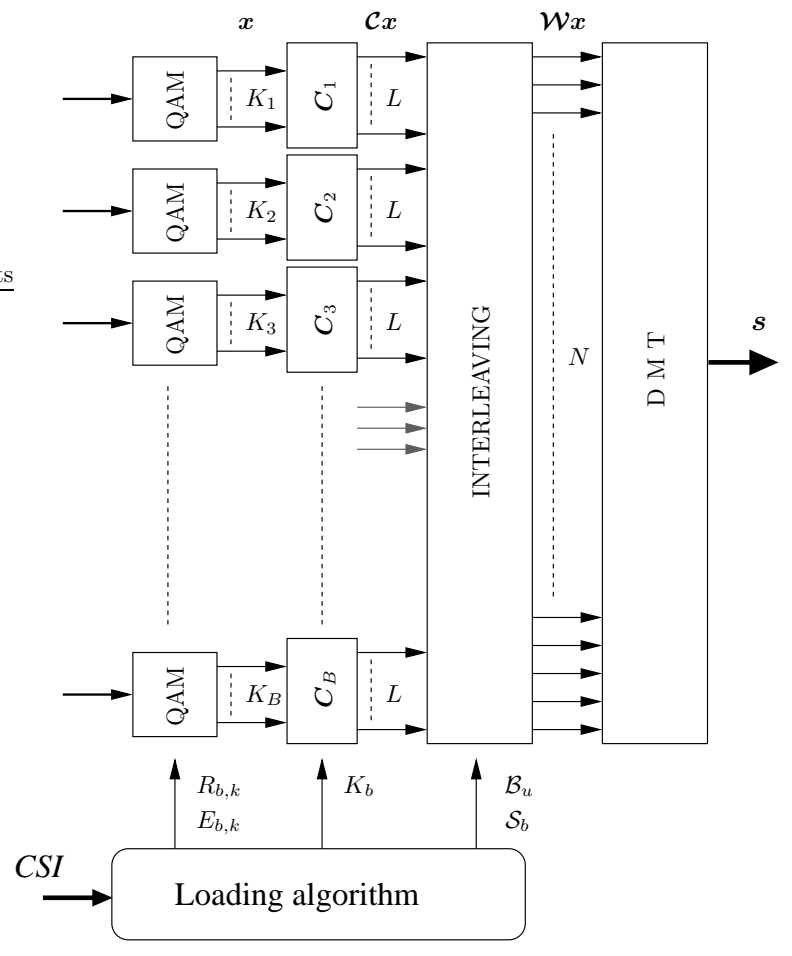

Figure 1: Proposed Adaptive SS-MC-MA transmitter

user and is consequently corrupted by a single channel, the allocation procedure is made easier, as we will understand later on.

The subcarrier subsets of the system are denoted $\mathcal{S}_{b}, b \in[1 ; B]$. We suppose that each subset clusters the same number $L$ of subcarriers, which implies that $B=$ $\left\lfloor\frac{N}{L}\right\rfloor$, where $N$ is the whole number of available subcarriers. The size of the subsets corresponds to the spreading factor, which means that $L$ is also the length of the spreading sequences to use within each subset. Note that in a general approach, each subset is not necessarly made of adjacent subcarriers. As each user can exploit several subsets, we also need to defi ne $\mathcal{B}_{u}$ the sets containing the indices $b$ of the subsets $\mathcal{S}_{b}$ collected by user $u, u \in[1 ; U]$.

The structure of the SS-MC-MA transmitter is depicted Fig. 1. A simple mathematical writing of the $N$ dimensional generated SS-MC-MA symbol vector $s$ is:

$$
s=\boldsymbol{F}^{H} \mathcal{W} \boldsymbol{x}
$$

with, $\mathcal{W}=\boldsymbol{Z} \cdot\left[\begin{array}{ccc}\boldsymbol{C}_{1} & & 0 \\ & \ddots & \\ 0 & & \boldsymbol{C}_{B}\end{array}\right]$ and, $\boldsymbol{x}=\left[\begin{array}{c}\boldsymbol{x}_{1} \\ \vdots \\ \boldsymbol{x}_{B}\end{array}\right]$.

Vectors $\boldsymbol{x}_{b}$ result from the serial to parallel conversion of the input data streams containing the $K_{b}$ QAM-mapped symbols to transmit within each subset. Like in digital subscriber line standards, M-QAM constellations of orders $m$ are used. Matrices $\boldsymbol{C}_{b}=\left\{c_{l, k}\right\}_{1 \leq l \leq L, 1 \leq k \leq K_{b}}$ are the individual spreading matrices bearing on their columns the $K_{b}$ sequences of length $L$ to use within each subset. In the 
following, orthogonal spreading sequences will be used, then $K_{b} \leq L, \forall b$. Matrix $Z$ is a permutation matrix that basically realizes an interleaving of the spread QAM symbols in the frequency domain. The spread and interleaved symbol vector is fi nally multiplied by the $N \times N$ Hermitian Fourier matrix $\boldsymbol{F}$ that realizes the multicarrier modulation. Invoking that matrices $C_{b}$ and $Z$ are unitary, it is straightforward to state that matrix $\mathcal{W}$ is also unitary.

To make the system adaptive, each $K_{b}$ spreading sequence of each subset $\mathcal{S}_{b}$ is associated to different modulation orders $R_{b, k}$ and different transmit energy levels $E_{b, k}$, $k \in\left[1 ; K_{b}\right]$. Moreover, the subcarriers are adaptively distributed among users. As shown in Fig 1, the loading algorithms proposed hereafter actually handle the optimal confi guration of the system, i.e. the optimal choice of all of these free parameters depending on a given optimization policy. Note that for $\mathcal{C}_{b}=\boldsymbol{I}_{L} \forall b$, where $\boldsymbol{I}_{L}$ denotes identity matrix of size $L \times L$, the system amounts to the classical DMT system combined with an FDMA component.

After classical cyclic prefi $x$ extension, the generated data stream is sent across the PLC channel. Assuming perfect synchronization and cyclic prefi $\mathrm{x}$ removal at the receiver, the channel can be modeled by one single complex coefficient per tone [12]. We then denote $\boldsymbol{H}=$ $\operatorname{diag}\left(h_{1}, \cdots, h_{N}\right)$ the $N \times N$ diagonal complex matrix whose diagonal terms are the frequency domain channel gains associated to each subcarrier $n \in[1 ; N]$. The channel effects are fi nally compensated for using a simple zeroforcing (ZF) equalizer before deinterleaving and despreading operations. Thus, the equalization matrix is diagonal and writes $\boldsymbol{H}^{-1}$. Since $\mathcal{W}$ is unitary, the vector of received data symbols $\boldsymbol{y}$ is

$$
\begin{aligned}
\boldsymbol{y} & =\mathcal{W}^{H} \boldsymbol{H}^{-1} \boldsymbol{H} \mathcal{W} \boldsymbol{x}+\mathcal{W}^{H} \boldsymbol{H}^{-1} \boldsymbol{n} \\
& =\boldsymbol{x}+\mathcal{W}^{H} \boldsymbol{H}^{-1} \boldsymbol{n},
\end{aligned}
$$

where vector $\boldsymbol{n}$ represents the additive white Gaussian noise such that $\mathrm{E}\left[\boldsymbol{n} \boldsymbol{n}^{H}\right]=\sigma_{n}^{2} \boldsymbol{I}_{N}$. Following the same formalism as in Eq. (1), we have $\boldsymbol{y}=\left[\boldsymbol{y}_{1} \ldots \boldsymbol{y}_{B}\right]^{T}$.

Note that a more powerful equalizer such as minimum mean square error (MMSE) equalizer could have been chosen to mitigate the noise effect. However, the use of a ZF detector leads to fairly simple manipulations resolving the different optimization problems detailed in the next sections. In fact, the mathematical expressions obtained with MMSE have a form such that the studied optimization problems can be shown to be almost untractable or leads to a prohibitive complexity.

\section{Problem Formulation}

In this section, the optimization problems studied in the paper are explicitly introduced in order to derive the loading algorithms in the following sections. We are interested in the maximization of both the throughput and the noise margin of each user of the system. Recall that the fundamental assumption is that the CSI is perfectly known at the transmitter and the receiver. The classical approach to state such optimization problems consists in computing the mutual information of the studied system. Assuming information vectors $\boldsymbol{x}$ and $\boldsymbol{y}$ as gaussian vectors, the mutual information of the SS-MC-MA system writes [13]

$$
I(\boldsymbol{x}|\boldsymbol{H}, \boldsymbol{y}| \boldsymbol{H})=\log \operatorname{det}\left(\boldsymbol{I}_{N}+\boldsymbol{R}_{\boldsymbol{x}, \boldsymbol{y}} \boldsymbol{R}_{\boldsymbol{y}, \boldsymbol{y}}^{-1}\right),
$$

where $\boldsymbol{R}_{\boldsymbol{x}, \boldsymbol{y}}$ and $\boldsymbol{R}_{\boldsymbol{y}, \boldsymbol{y}}$ are covariance matrices of size $K_{b} \times$ $K_{b}$ that respectively write $\mathrm{E}\left[\boldsymbol{x} \boldsymbol{y}^{H}\right]$ and $\mathrm{E}\left[\boldsymbol{y} \boldsymbol{y}^{H}\right]$. From the structure of matrix $\mathcal{W}$ one can verify that

$$
I(\boldsymbol{x}|\boldsymbol{H}, \boldsymbol{y}| \boldsymbol{H})=\log \prod_{b=1}^{B} \operatorname{det}\left(\boldsymbol{I}_{L}+\boldsymbol{R}_{\boldsymbol{x}_{b}, \boldsymbol{y}_{b}} \boldsymbol{R}_{\boldsymbol{y}_{b}, \boldsymbol{y}_{b}}^{-1}\right),
$$

with $\boldsymbol{R}_{\boldsymbol{x}_{b}, \boldsymbol{y}_{b}}=\mathrm{E}\left[\boldsymbol{x}_{b} \boldsymbol{y}_{b}^{H}\right]$ and $\boldsymbol{R}_{\boldsymbol{y}_{b}, \boldsymbol{y}_{b}}=\mathrm{E}\left[\boldsymbol{y}_{b} \boldsymbol{y}_{b}^{H}\right]$. After simple mathematical derivations, we obtain

$$
\begin{aligned}
& \boldsymbol{R}_{\boldsymbol{x}_{b}, \boldsymbol{y}_{b}}=\boldsymbol{R}_{\boldsymbol{x}_{b}, \boldsymbol{x}_{b}}+\frac{\sigma_{n}^{2}}{L} \sum_{l \in \mathcal{S}_{b}} \frac{1}{\left|h_{l}\right|^{2}} \boldsymbol{I}_{L}, \\
& \boldsymbol{R}_{\boldsymbol{y}_{b}, \boldsymbol{y}_{b}}=\boldsymbol{R}_{\boldsymbol{x}_{b}, \boldsymbol{x}_{b}} .
\end{aligned}
$$

Substituting (5) and (6) in (4), it comes,

$$
\begin{aligned}
& I(\boldsymbol{x}|\boldsymbol{H}, \boldsymbol{y}| \boldsymbol{H})= \\
& \quad \log \prod_{b=1}^{B} \operatorname{det}\left(\boldsymbol{I}_{L}+\frac{L}{\sum_{l \in \mathcal{S}_{b}} \frac{1}{\left|h_{l}\right|^{2}}} \frac{1}{\sigma_{n}^{2}} \boldsymbol{R}_{\boldsymbol{x}_{b}, \boldsymbol{x}_{b}}\right) .
\end{aligned}
$$

Eventually, all the matrices in (7) are diagonal which allows to rapidly compute the determinant. Denoting $\sigma_{x_{b, k}}^{2}, k \in\left[1 ; K_{b}\right]$ the diagonal elements of matrix $\boldsymbol{R}_{\boldsymbol{x}_{b} \boldsymbol{x}_{b}}$, i.e. the amounts of energy associated to each QAM symbol transmitted in the subset $\mathcal{S}_{b}$, we have

$$
I(\boldsymbol{x}|\boldsymbol{H}, \boldsymbol{y}| \boldsymbol{H})=\sum_{b=1}^{B} \sum_{k=1}^{K_{b}} \log \left(1+\frac{L}{\sum_{l \in \mathcal{S}_{b}} \frac{1}{\left|h_{l}\right|^{2}}} \frac{\sigma_{x_{b, k}}^{2}}{\sigma_{n}^{2}}\right) .
$$

Due to spreading, the amount of energy $\sigma_{x_{b, k}}^{2}$ is equally distributed across the chips of the spreading sequences. $\sigma_{x_{b, k}}^{2}$ can then be expressed as $L \cdot E_{b, k}$, according to the notation introduced in the previous section. Denoting $R_{b}$ the throughput reached in subset $\mathcal{S}_{b}$, the achievable rate $R_{u}$ per user expresses

$$
\begin{aligned}
R_{u} & =\sum_{b \in \mathcal{B}_{u}} R_{b}=\sum_{b \in \mathcal{B}_{u}} \sum_{k=1}^{K_{b}} R_{b, k} \\
& =\sum_{b \in \mathcal{B}_{u}} \sum_{k=1}^{K_{b}} \log _{2}\left(1+\frac{1}{\gamma_{u} \Gamma} \frac{L^{2}}{\sum_{l \in \mathcal{S}_{b}} \frac{1}{\left|h_{l, u}\right|^{2}}} \frac{E_{b, k}}{\sigma_{n}^{2}}\right),
\end{aligned}
$$


where $h_{l, u}$ denotes the channel gain of user $u$ 's subcarrier $l$. $\Gamma$ is the SNR gap and $\gamma_{u}$ is the noise margin gap of user $u$. As defi ned in [8], $\Gamma$ is a measure of the loss introduced by the QAM with respect to capacity. This gap allows the system to garantee a given target symbol error rate (SER) using a discrete QAM symbol source instead of a continuous gaussian source. For modulation orders $m \leq 2$, a convenient approximation leads to an SNR gap $\Gamma$ independent of $m$ [8]. We then exclude $m=1$ from the possible orders and choose $m \in[2 ; 15]$ as in DSL. Noise margin $\gamma_{u}$ is an additional gap that increases the robustness of the system against noise levels higher than expected.

For a given system, two possible optimization policies can be followed: either the throughput is maximized for a given noise margin, or the noise margin is maximized for a given target throughput. In the sequel, both optimization problems are studied for the SS-MC-MA multiuser system. More precisely, we propose to maximize individual throughputs $R_{u}$ (resp. noise margin $\gamma_{u}$ ) rather than to maximize the total thoughput (resp. the global noise margin) of the system. Such an approach maximizes the minimal throughput (resp. noise margin) among users and allows to ensure a quality of service for each user. Hence, the maximization problems can be stated as

$$
\begin{array}{ll}
(P 1): \quad \forall u, \max _{\mathcal{B}_{u}, \mathcal{S}_{b}, K_{b}, E_{b, k}} R_{u}, \\
\text { subject to } \quad \begin{cases}(C 1 a) & \forall u \neq u^{\prime} \mathcal{B}_{u} \cap \mathcal{B}_{u^{\prime}}=\emptyset \\
(C 1 b) & \forall b \neq b^{\prime} \mathcal{S}_{b} \cap \mathcal{S}_{b^{\prime}}=\emptyset \\
(C 1 c) & \forall b K_{b} \leq L \\
(C 1 d) & \forall b, k R_{b, k} \in[2 ; 15] \\
(C 1 e) & \forall b \sum_{k}^{K_{b}} E_{k, b} \leq \check{E}\end{cases} \\
\text { subject to } \quad \begin{cases}\left.\max _{u}\right): \\
\mathcal{B}_{u}, \mathcal{S}_{b}, K_{b}, E_{b, k} \\
(C 2 a) & \forall u \neq u_{u}^{\prime} \mathcal{B}_{u} \cap \mathcal{B}_{u^{\prime}}=\emptyset \\
(C 2 b) & \forall b \neq b^{\prime} \mathcal{S}_{b} \cap \mathcal{S}_{b^{\prime}}=\emptyset \\
(C 2 c) & \forall b K_{b} \leq L \\
(C 2 d) & \forall b, k R_{b, k} \in[2 ; 15] \\
(C 2 e) & \sum R_{b}=\check{R}\end{cases}
\end{array}
$$

Both optimization problems are based on the same constraints, except for constraints $(C 1 e)$ and $(C 2 e)$. Constraints $(C 1 a)$ or $(C 2 a)$ combined with constraints $(C 1 b)$ or $(C 2 b)$ ensure that the users can neither share the same subset nor share the same subcarriers, which garantees FDMA. Constraints $(C 1 c)$ or $(C 2 c)$ expresses the limitation due to the use of orthogonal spreading sequences. Constraints $(C 1 d)$ or $(C 2 d)$ comes from the QAM modulation order limitation. As for the throughput maximization problem, $(C 1 e)$ corresponds to the PSD constraint, where $\check{E}$ is the maximum allowed PSD level. Concerning the noise margin maximization problem, $(C 2 e)$ is the target rate constraint, denoted $\check{R}$.
Each optimization problem $(P 1)$ and $(P 2)$ can be decomposed into two independent maximization tasks. One can first assume an arbitrary subcarrier sharing among users and find an allocation procedure leading to maximal throughput (resp. noise margin) over the created subsets. Exploiting the obtained loading strategy, the subcarrier sharing problem can be worked out in a second time. In the following sections, we will derive the solution to each optimization problem and propose practical loading algorithms according to this problem decomposition.

\section{ThroughPUT MAXIMIZATION}

In this section, we establish a specifi c proposition that give the optimal loading strategy to use in order to maximize the throughput of each user $u$ who have been arbitrary assigned some subcarrier subsets. Hence, we aim at solving the following problem

$$
\forall u, \max _{K_{b}, E_{b, k}} R_{u}, \text { subject to }(C 1 c),(C 1 d),(C 1 e),
$$

where $R_{u}$ is given by (9) with $\mathcal{B}_{u}$ and $\mathcal{S}_{b}$ already fi xed. For a given user $u$, this problem can be straightforwardly restated as

$$
\forall b, \max _{K_{b}, E_{b, k}} R_{b} \text {, subject to }(C 1 c),(C 1 d),(C 1 e),
$$

since the total throughput $R_{u}$ achieved by user $u$ over its subsets is maximized when the throughputs achieved within each of these subsets are individually maximized. As throughput maximization is considered, we set $\gamma_{u}=1$ hereafter.

\subsection{INFINITE GRANULARITY CASE}

If constraint $(C 1 d)$ is lifted, the use of continuous modulation orders are allowed, i.e. $R_{b, k} \in \mathbb{R}_{+}$. This theoritical situation will be referred to as the infi nite granularity case (IG) throughout the paper. In that case, classical Lagrange optimization applied to $R_{b}$ leads to a fairly simple solution which consists in achieving a uniform distribution of bits and energy between the $L$ available codes in subset $\mathcal{S}_{b}$ [14]. More precisely, optimal energy and bit distribution is achieved when $\forall k, E_{k, b}^{*}=\check{E} / L$ and $R_{k, b}^{*}=R_{b}^{*} / L$, where superscript * denotes optimality. Assuming $\check{E}=1$ without loss of generality, $R_{b}^{*}$ is hence obtained substituting $E_{k, b}$ with $E_{k, b}^{*}$ in (9),

$$
R_{b}^{*}=L \log _{2}\left(1+\frac{1}{\Gamma} \frac{L}{\sigma_{n}^{2} \sum_{l \in \mathcal{S}_{b}} \frac{1}{\left|h_{l, u}\right|^{2}}}\right) .
$$


Note that this result implicitly says that all of the available codes must be exploited to ensure maximal throughput.

\subsection{Finite GRANULARITY CASE}

When fi nite order modulations are used, which corresponds to the fi nite granularity (FG) case, the Lagrange solution can not be applied and the problem gets a bit trickier. However, from the Lagrange solution we intuitively expect that the optimal distribution of bits between the codes should be as uniform as possible. This means that among the $L$ available codes, some $n$ codes should receive $q+1$ bits and $L-n$ codes should receive $q$ bits, with $q, n \in \mathbb{N}$. To verify this intuitive assumption, let us show that such a distribution minimizes the energy cost. From (9), the energy cost $E_{b}$ within a given subset $\mathcal{S}_{b}$ can be expressed as

$$
E_{b}=\sum_{k=1}^{L} E_{b, k}=\frac{1}{\alpha} \sum_{k=1}^{L}\left(2^{R_{b, k}}-1\right)
$$

with $\alpha=\frac{1}{\Gamma \sigma_{n}^{2}} \frac{L^{2}}{\sum_{l \in \mathcal{S}_{b}} \frac{1}{\left|h_{l, u}\right|^{2}}}$.

Assuming that bits are initially distributed following the intuitively stated strategy, we just have to check that any bit exchange between any codes leads to an increase of the energy cost. All of the possible exchanges can actually be summarized into four particular cases which are

$$
\begin{aligned}
& \left\{\begin{array} { l } 
{ R _ { i } = q \mapsto q + a } \\
{ R _ { j } = q \mapsto q - a }
\end{array} \quad \left\{\begin{array}{l}
R_{i}=q+1 \mapsto q+1+a \\
R_{j}=q+1 \mapsto q+1-a
\end{array}\right.\right. \\
& \left\{\begin{array} { l } 
{ R _ { i } = q \mapsto q + a } \\
{ R _ { j } = q + 1 \mapsto q + 1 - a }
\end{array} \quad \left\{\begin{array}{l}
R_{i}=q+1 \mapsto q+1+a \\
R_{j}=q \quad \mapsto q-a
\end{array}\right.\right.
\end{aligned}
$$

where $a$ is the number of exchanged bits. For the fi rst case, the cost difference $\Delta E$ between the initial and the modifi ed bit distributions gives

$$
\begin{aligned}
\Delta E= & (L-n-2) 2^{q}+n 2^{q+1}+2^{q+a}+2^{q-a} \\
& -(L-n) 2^{q}-n 2^{q+1} \\
= & 2^{q-a}\left(2^{a+1}\left(2^{a-1}-1\right)+1\right) .
\end{aligned}
$$

Since $a \geq 1$, we have $\Delta E>0$, which means that the new distribution is more expensive than the initial one. Following the same analysis for the other three cases leads to similar conclusions. We then conclude that the proposed bit distribution is optimal in the sense of energy cost.

In order to totaly defi ne the distribution strategy, it remains to fi nd adequate $q$ and $n$ that maximize the throughput while respecting the PSD constraint. From previous section, it is known that maximal throughput with IG is $R_{b}^{*}$. It is then clear that assigning $\left\lfloor R_{b}^{*} / L\right\rfloor$ bits to each code leads to an energy cost inferior to the PSD limitation. On the other hand, assigning $\left\lfloor R_{b}^{*} / L\right\rfloor+1$ bits to each code violate the PSD constraint. We then deduct that $q=\left\lfloor R_{b}^{*} / L\right\rfloor$.
Parameter $n$ can hence simply be obtained solving the following inequation

$$
\begin{aligned}
\check{E}-E_{b}= & \frac{L}{\alpha}\left(2^{R_{b}^{*} / L}-1\right)-\frac{n}{\alpha}\left(2^{\left\lfloor R_{b}^{*} / L\right\rfloor+1}-1\right) \\
& -\frac{L-n}{\alpha}\left(2^{\left\lfloor R_{b}^{*} / L\right\rfloor}-1\right) \geq 0 .
\end{aligned}
$$

The greatest $n \in \mathbb{N}$ that is solution of this inequality is

$$
n=\left\lfloor L\left(2^{R_{b}^{*} / L-\left\lfloor R_{b}^{*} / L\right\rfloor}-1\right)\right\rfloor .
$$

Note that the obtained bit distribution not necessarily leads to a full load SS-MC-MA system, i.e. $\forall b K_{b}=L$, since parameter $q$ can be equal to zero. In fact, the effective number of used codes within a given subset $\mathcal{S}_{b}$ writes $K_{b}^{*}=q+n$ and is maximal if and only if $R_{b}^{*} \geq L$.

These derivations fi nally give the optimal bit allocation strategy to follow to maximize the throughput within a given subcarrier subset. Applying this strategy to the whole SS-MC-MA system gives the optimal bit and energy distribution that maximizes the throughput of each user for a given subcarrier distribution. We can then state the following proposition.

Proposition 1 Let denote $\mathcal{R}$ the bit allocation policy. For a given subset allocation among users, the throughput of the system is maximized if, in each subset $\mathcal{S}_{b}, \mathcal{R}$ assigns $\left(\left\lfloor R_{b}^{*} / L\right\rfloor+1\right)$ bits to $n_{b}$ codes and $\left\lfloor R_{b}^{*} / L\right\rfloor$ bits to $\left(L-n_{b}\right)$ codes, where $n_{b}=\left\lfloor L\left(2^{R_{b}^{*} / L-\left\lfloor R_{b}^{*} / L\right\rfloor}-1\right)\right\rfloor$. The achieved rates are denoted $\bar{R}_{k, b}^{*}$ and the related energies express

$$
\bar{E}_{k, b}^{*}=\frac{\Gamma}{L^{2}} \sum_{l \in \mathcal{S}_{b}} \frac{\sigma_{n}^{2}}{\left|h_{l, u}\right|^{2}}\left(2^{\bar{R}_{k, b}^{*}-1}\right) .
$$

Eq. (19) is simply obtained substituting $\bar{R}_{k, b}^{*}$ in (9). The rate in each subset $\mathcal{S}_{b}$ reaches the value $\bar{R}_{b}^{*}=$ $\sum_{k} \bar{R}_{k, b}^{*}$, and the maximal rate of user $u$ writes $\bar{R}_{u}^{*}=$ $\sum_{b} \bar{R}_{b}^{*}$. Hence, the throughput maximization is fairly simple and requires, for each subset, the computation of $R_{b}^{*}$, $n_{b}$ and of only two values of rates $\bar{R}_{k, b}^{*}$ and energies $\bar{E}_{k, b}^{*}$. Finally, it is interesting to note that for $L=1$, the proposed allocation policy amounts to classical bit loading of the DMT. In that case $n_{b}=1$, and one value of rate $\left\lfloor\bar{R}_{b}^{*}\right\rfloor$ and energy $\bar{E}_{b}^{*}$ have to be computed for each subcarrier, which turns out more expensive than with SS-MC-MA.

\section{NOISE MARGIN MAXIMIZATION}

Let us now focus on the noise margin maximization problem for each user $u$ of the system, assuming an arbitrary distribution of the subcarriers between users. This maximization problem then expresses

$$
\max _{K_{b}, E_{b, k}} \gamma_{u}, \text { subject to }(C 2 c),(C 2 d),(C 2 e) \text {. }
$$


As evident from equation (9), no simple closed form solution can be derived to obtain the noise margin $\gamma_{u}$ for a target throughput $R_{u}$. This implies that no analytical solution exists to this maximization problem and that an iterative algorithm will be required to reach the optimal solution. Let us restate the problem as

$$
\forall b, \max _{K_{b}, E_{b, k}} \gamma_{b}, \text { subject to }(C 2 c),(C 2 d),(C 2 e),
$$

where $\gamma_{b}$ is the achieved noise margin in subset $\mathcal{S}_{b}$. Solving this problem amounts to find the optimal sharing of the target rate $\check{R}$ between the available subsets $\mathcal{S}_{b}$ in order to jointly maximize each individual noise margin $\gamma_{b}$. In the sequel, we will fi rst establish the loading strategy that leads to maximal noise margins $\gamma_{b}$ for given target rates $\check{R}_{b}$ within each subset $\mathcal{S}_{b}$. Then, the optimal distribution of the target rate $\check{R}$ between the subsets will be studied. As noise margin maximization is considered, we set $E_{b}=\check{E}$ hereafter.

\subsection{INDIVIDUAL SUBSET NOISE MARGIN MAXI- MIZATION}

The problem here is to fi nd the optimal loading procedure in the sense of the noise margin maximization, to distribute $\check{R}_{b}$ bits between the $L$ available codes of each subset $\mathcal{S}_{b}$. Equivalently, we have to propose a bit distribution strategy within each subset in order to minimize the energy cost and for target rate $\check{R}_{b}$. This problem has actually been solved in section 4 , under the intuitive idea of a bit distribution as uniform as possible. Applying the obtained results for a target rate $\check{R}_{b}, q$ becomes equal to $\left\lfloor\check{R}_{b} / L\right\rfloor$. Parameter $n$ is then the solution of the following equation

$$
n\left(\left\lfloor\check{R}_{b} / L\right\rfloor+1\right)+(L-n)\left\lfloor\check{R}_{b} / L\right\rfloor=\check{R}_{b},
$$

which leads to $n=\check{R}_{b}-L\left\lfloor\check{R}_{b} / L\right\rfloor$. As mentioned is the previous section, the number of used codes within the considered subset is maximal if and only if the target rate $\check{R}_{b}$ for this subset is higher than the spreading factor $L$.

From these considerations, the optimal allocation policy is then known and we can state the following proposition.

Proposition 2 Let denote $\mathcal{R}$ the bit allocation policy. For a given subset allocation among users and given individual target rates $\breve{R}_{b}$, the noise margin of the system is maximized if, in each subset $\mathcal{S}_{b}, \mathcal{R}$ assigns $\left(\left\lfloor\check{R}_{b} / L\right\rfloor+1\right)$ bits to $\left(\check{R}_{b}-\right.$ $\left.\left\lfloor\check{R}_{b} / L\right\rfloor L\right)$ codes and $\left\lfloor\check{R}_{b} / L\right\rfloor$ bits to $L-\left(\check{R}_{b}-\left\lfloor R_{b} / L\right\rfloor L\right)$ codes. The achieved rates are denoted $\bar{R}_{b, k}^{*}$ and the related energies are

$$
\bar{E}_{b, k}^{*}=\frac{2^{\bar{R}_{b, k}^{*}-1}}{\sum_{k=1}^{L}\left(2^{\bar{R}_{b, k}^{*}}-1\right)} \check{E} .
$$

Equation (23) is obtained from (9) and using $\sum_{k} \bar{E}_{b, k}=\check{E}$. The optimal achieved noise margin on subset $\mathcal{S}_{b}$ then writes

$$
\bar{\gamma}_{b}^{*}=\frac{1}{\sum_{k}^{L} 2^{\bar{R}_{b, k}^{*}-1}} \frac{1}{\Gamma} \frac{L^{2}}{\sum_{l \in \mathcal{S}_{b}} \frac{1}{\left|h_{l, u}\right|^{2}}} \frac{\check{E}}{\sigma_{n}^{2}} .
$$

Hence, the individual noise margin maximization is simple since it requires the only computation of $n_{b}$ and of two values of rates $\bar{R}_{k, b}^{*}$ and energies $\bar{E}_{k, b}^{*}$.

\subsection{JOINT NOISE MARGIN MAXIMIZATION}

Prop. 2 assumes that individual target rates $R_{b}$ for each subset $\mathcal{S}_{b}$ are known. As target rates $\check{R}=\sum_{b} R_{b}$ rather than individual $\check{R}_{b}$ are specifi ed, the loading algorithm must handle the rate distribution among the subsets while jointly maximizing the noise margin in each subset $\mathcal{S}_{b}$. The optimization problem (21) can be restated as

$$
\max _{\widetilde{R}_{b}} \min _{b} \bar{\gamma}_{b}^{*} \text {, subject to }(C 2 c),(C 2 d),(C 2 e) .
$$

Since the noise margin $\bar{\gamma}_{b}^{*}$ resulting from the assignment of a certain number of bits in a subset is independent of the numbers of bits assigned to other subsets, it turns out that a greedy approach is optimal to solve this problem [15]. The basic idea is to iteratively assign bits to the subsets, one bit at a time, and at each iteration, an additional bit is assigned to the subset that will exhibit the highest noise margin $\gamma_{b}$ after receiving one more bit. The loading procedure can then be described as follows.

$$
\begin{aligned}
& \text { 1. Initialization. } \\
& \text { a. Set } \forall b \quad R_{b}=0 \text {. } \\
& \text { b. Compute } \forall b \bar{\gamma}_{b}^{*} \text { considering } R_{b}+1 \\
& \text { 2. While } \sum_{b} R_{b}<R_{u} \\
& \text { a. Find } b=\arg \min _{b}\left(\bar{\gamma}_{b}^{*}\right) \text {. Assign one more } \\
& \text { bit to the selected subset } \mathcal{S}_{b} \text {, } \\
& \text { c. Update } R_{b}=R_{b}+1 \text { for the found } b \text { and } \\
& \text { compute new } \bar{\gamma}_{b}^{*} \text { for } R_{b}+1
\end{aligned}
$$

Note that this greedy procedure is very similar to the well-known algorithm proposed in [9]. However, the main constraint in [9] is based on a total energy cost limitation, and not on a PSD limitation as considered herein. The resulting algorithm is therefore different. Moreover, as we consider that subcarriers are clustered into subsets and bound by spreading sequences, each additionnal bit assignment in the above stated algorithm must be processed with respect to Prop. 2. More precisely, this means that each additional bit is assigned to one of the codes among those of the selected subset that bear the lower number of bits. The presented algorithm is a bit-additive algorithm, with an initialization state $\forall b R_{b}=0$. A bit-removal approach could 
also be led starting from the state $\forall b R_{b}=\bar{R}_{b}^{*}$, without changing the fi nal allotment result.

Finally, one can verify that the proposed allocation policy remains valid for $L=1$, and corresponds to the margin maximization task for the particular case of DMT. Similarly to the throughput maximization, complexity increases in that case, since the number of subsets, and thus of rates and energies to compute, becomes maximal.

\section{ADAPTIVE SUBCARRIER DISTRIBUTION ALGORITHMS}

In the previous sections, both optimization procedures consider that the sharing of the spectrum between the users has already been processed. The subcarrier distribution policy has to be studied in order to optimize the spectrum resource. From the above obtained results, the maximization problems can be restated as

$$
\begin{aligned}
& (P 1): \max _{\left\{\mathcal{S}_{b}, \mathcal{B}_{u}\right\}}\left(\min _{u} \bar{R}_{u}^{*}\right), \\
& (P 2): \max _{\left\{\mathcal{S}_{b}, \mathcal{B}_{u}\right\}}\left(\min _{u} \bar{\gamma}_{u}^{*}\right) .
\end{aligned}
$$

These are classical max-min problems and the maximization task essentially consists in fi nding the different subsets so that each user can maximize its specifi c metric. Let us denote $\eta_{u}$ this metric. For throughput maximization problem $(P 1), \eta_{u}$ is the rate $\bar{R}_{u}^{*}$ assigned to user $u$, and for margin maximization $(P 2), \eta_{u}$ is the margin $\bar{\gamma}_{u}^{*}$ of user $u$. To fi nd the optimal solution, each problem should be formulated into a standard convex optimization problem. However, as the resulting algorithm would require a prohibitive intensive computation, a sub-optimal but fairly simple solution is proposed as in [16]. The solution consists in iteratively assigning one block at a time to the user $u$ that benefi ts from the lowest metric $\eta_{u}$. In order to increase $\eta_{u}$ as much as possible, each new block should be made up of the best available subcarriers. The obtained subcarrier distribution is the optimal solution in the case of FG [14]. After each assignment, the metric value is updated with respect to the previously stated propositions, namely Proposition 1 for problem $(P 1)$ or Proposition 2 for problem $(P 2)$. A priority order among user is required to allocate a fi rst block to each user and provide initial individual metric values. A convenient solution is to sort the users by ascending order of channel response amplitude. The detailed algorithm writes as follows.

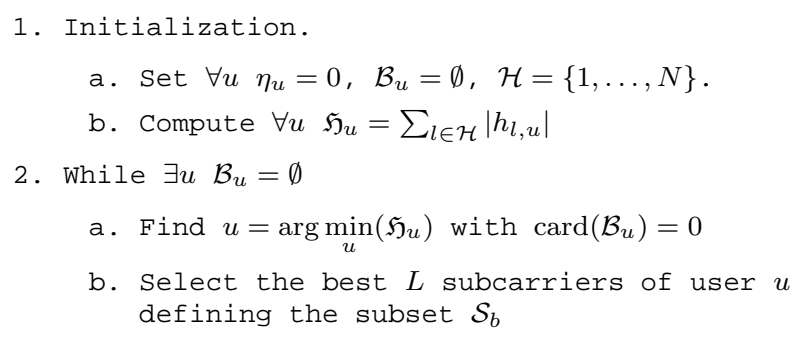

$$
\text { c. Compute } \eta_{u} \text {. Update } \mathcal{H}=\mathcal{H}-\mathcal{S}_{b}, \mathcal{B}_{u}=\{b\}
$$

3. While $\mathcal{H} \neq \emptyset$

$$
\begin{aligned}
& \text { a. Find } u=\arg \min _{u}\left(\eta_{u}\right) \\
& \text { b. Select the best } L \text { subcarriers of user } u \\
& \text { defining a new subset } \mathcal{S}_{b} \\
& \text { c. Compute new } \eta_{u} \text {. Update } \mathcal{H}=\mathcal{H}-\mathcal{S}_{b}, \mathcal{B}_{u}= \\
& \mathcal{B}_{u}+\{b\}
\end{aligned}
$$

The proposed iterative algorithm actually consists in a greedy procedure applied to the subcarrier distribution. As an FDMA approach is carried out, assigning a subcarrier to a particular user prevents other users from using that subcarrier. This dependency makes any greedy algorithm suboptimal [15]. Nevertheless, we will see in simulations that the proposed scheme offers very satisfying results.

As already mentioned in section 2, a ZF dectector has been selected instead of an MMSE detector or a more effi cient one. Beyond the already stated complexity arguments in favor of $\mathrm{ZF}$, note that the subcarrier distribution minimizes the channel distorsion within each subset. This makes ZF near optimal since ZF and MMSE performances converge in flat fading channels.

\section{Simulation RESUlts}

We applied the proposed algorithms to the case of a 4user multiple access communication over the channels presented Fig. 2. Channel responses have been measured in an outdoor powerline network by the French power supply company (EDF). The generated multicarrier signal is composed of $N=1880$ usefull subcarriers transmitted in the band $1.6-20 \mathrm{MHz}$, which is the suggested band for outdoor PLC. The intercarrier spacing is $9.765 \mathrm{kHz}$ and a guard interval of $5 \mu$ s is employed to avoid inter-symbol interference. As already mentioned, we use the $2^{m}$-ary QAM constellations specifi ed for DSL, i.e. $m \in[2 ; 15]$. Moreover,

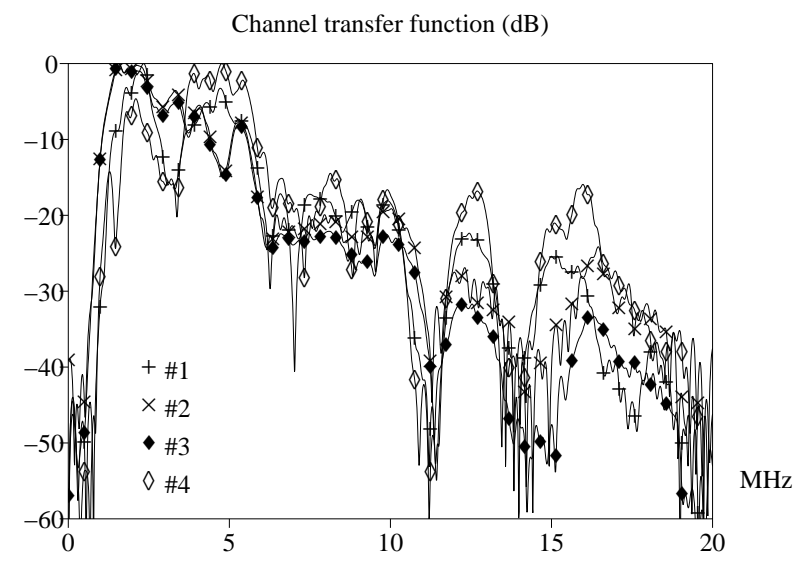

Figure 2: Examples of measured PLC channel responses 

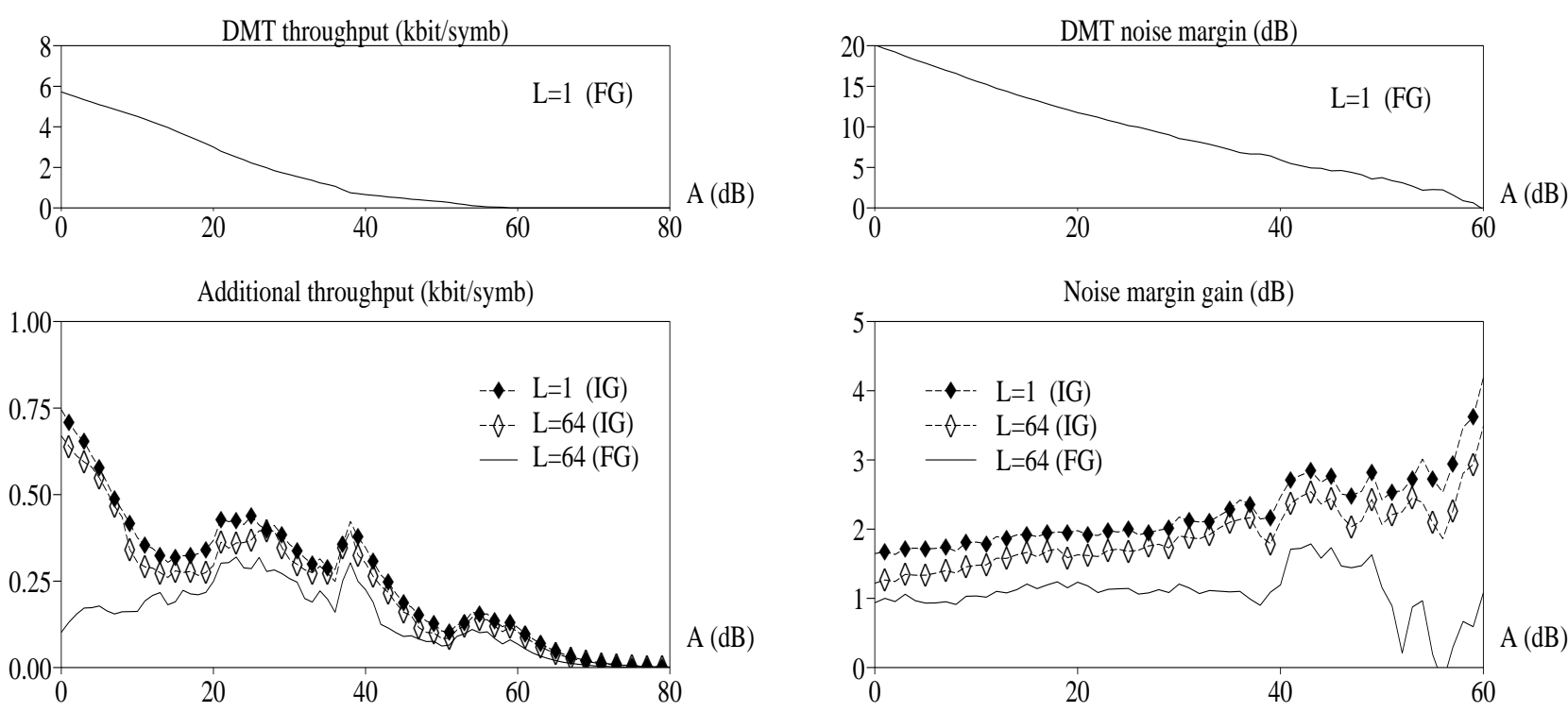

Figure 3: Achieved throughputs for the proposed adaptive SS$M C-M A$ system relatively to DMT

Figure 4: Achieved margins for the proposed adaptive SS-MCMA system relatively to DMT

it is supposed that synchronization and channel estimation tasks have been processed successfully. We also recall that perfect CSI is available at both the transmitter and the receiver. The performance of the new system are compared to those obtained with DMT. Note that DMT is equivalent to the proposed SS-MC-MA system with $L=1$. We assume a background noise level at $-110 \mathrm{dBm} / \mathrm{Hz}$ [3] and a flat transmission PSD of $-40 \mathrm{dBm} / \mathrm{Hz}$. Results are given for a target SER of $10^{-3}$ without channel coding, corresponding to an SNR gap $\Gamma=6 \mathrm{~dB}$ [8].

Fig. 3 shows the results obtained when the system is confi gured to maximize the throughput. Results are given versus parameter $A_{d B}$ which conveys the attenuation experienced by the signal through the channel. From the chosen transmission and noise levels, this implies for example that above $A=70 \mathrm{~dB}$, all of the subcarriers have individual SNR lower than $0 \mathrm{~dB}$. The system performance is presented in the case of infi nite and fi nite granularity, respectively denoted IG and FG, and for a spreading factor $L=64$ with SS-MC-MA and $L=1$ corresponding to DMT. As we aim at comparing the performance of both systems, results are displayed relatively to the DMT with FG, as evident from Fig. 3. The DMT reference curve is displayed in the top fi gure and the given results are average throughputs per user, expressed in kbit per multicarrier symbol.

We first verify that results are always better with IG than with FG for both systems, which is the direct consequence of the quantifi cation loss mentioned in the introduction. Concerning the results with IG, we note that DMT outperforms SS-MC-MA for all of the attenuations. This behavior could actually be expected since the combination of DMT with adaptive modulations is known as the optimal solution when no restrictions are imposed on the modulation orders. SS-MC-MA gives slightly lower rates with IG,

which is due to the use of a simple ZF detector.

It is interesting to focus on the results obtained with FG, which is the practical transmission scenario. Due to the quantifi cation loss, SS-MC-MA achieve lower throughputs with FG than with IG, but exhibits higher throughputs than DMT in that case. For example, for an attenuation of $40 \mathrm{~dB}$, SS-MC-MA can transmit an additionnal throughput of about $215 \mathrm{bit} / \mathrm{symb}$ per user compared to DMT. This additionnal throughput corresponds to a gain of $2 \mathrm{Mbit} / \mathrm{s}$ per user and a total gain of about $8 \mathrm{Mbit} / \mathrm{s}$. Using the reference results of the top curve, the corresponding total throughputs are around $48 \mathrm{Mbps}$ for SS-MC-MA against only $40 \mathrm{Mbps}$ for DMT. The important result is then that SS-MC-MA performs closer to the IG upper-bound than DMT, which means that SS-MC-MA is more effi cient in ressource allocation than DMT. This behavior can be explained by the energy gathering capability of SS-MC-MA within each subcarrier block. Contrary to DMT, the proposed system can advantageously collect and exploit the residual energies lost on each subcarrier of the DMT system because of the fi nite granularity of the QAM modulations. Consequently, the proposed system better exploits the energy resource and yields signifi cant throughput improvements. From the results of fi gure 3, one can however note that the throughput gain is less signifi cative for low attenuations. This saturation is in fact simply due to the modulation order limitation.

If we now run the noise margin algorithm, we obtain the results presented in Fig. 4. The display formalism is the same than the one used in Fig. 3. For each attenuation $A$, the specifi ed target rate corresponds to the half of the maximal achievable rate, i.e. the half of the thoughput achieved with infi nite granularity when $L=1$. General observations are similar to those mentioned in the throughput 

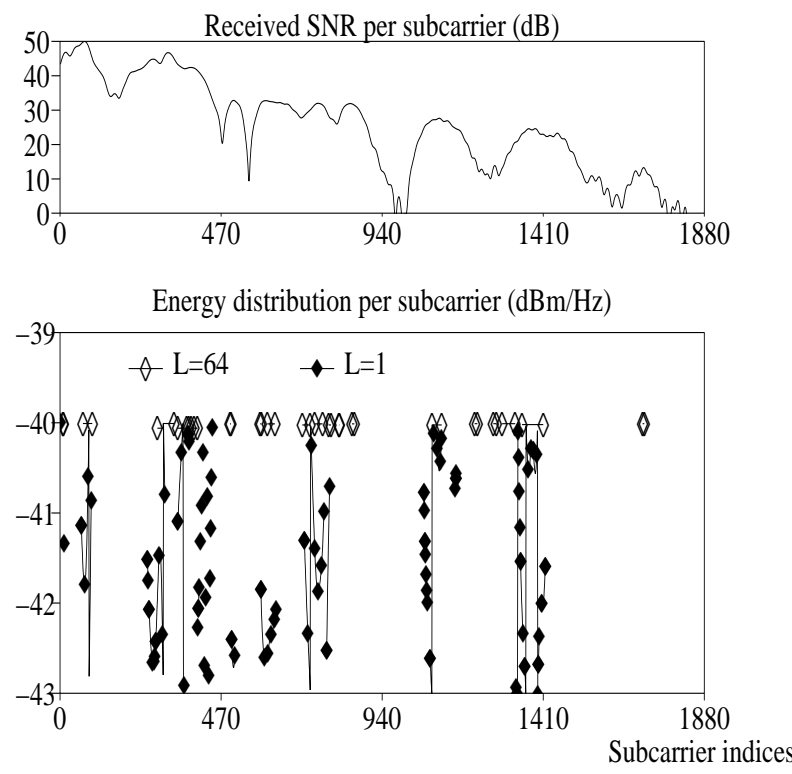

Figure 5: Comparison of the energy allocation between the proposed adaptive SS-MC-MA system and DMT

maximization case. As evident from the plotted curves, the proposed scheme is indeed able to perform very close to the IG upper limit and outperforms the DMT system with FG. For instance, for attenuations less than $50 \mathrm{~dB}$, the exhibited noise margin with SS-MC-MA is around $1 \mathrm{~dB}$ higher than with DMT. These results indicate that the transmitted bits are better distributed across the spectrum when spreading is processed. Recall that any noise margin increase improves the robustness of the system, which is worth of interest in a transmission context as noisy as the PLC's.

To emphasize the fact that SS-MC-MA allows to better exploit the energy resource, let us analyze Fig. 5. In this fi gure, we present the results obtained in terms of energy allocation per subcarrier in the case of throughput maximization for a given user among 4 . The top fi gure represents the received SNR per subcarrier in the case of a full energy transmission, i.e. the DSP of the transmitted signal has a flat level of $-40 \mathrm{dBm} / \mathrm{Hz}$. The energy distribution exhibits variations of $3 \mathrm{~dB}$ with DMT $(L=1)$, whereas this distribution is almost flat with SS-MC-MA $(L=64)$. Note that the used subcarriers are spread over the whole spectrum, but that each system does not always exploit the same sets of subcarriers for the given user. Moreover, due to the spread spectrum component, the SS-MC-MA system is able to exploit subcarriers with lower individual SNR than DMT does. SS-MC-MA is then more effi cient in exploiting the available energy.

\section{Conclusion}

In this paper, a new system has been proposed, combining adaptive resource allocation principles with the SSMC-MA waveform. Loading procedures have been devel- oped as a solution to the throughput and the noise margin maximization problem for this new adaptive SS-MC-MA system, under PSD and fi nite order modulation constraints. A practical subcarrier sharing algorithm has also been introduced to either maximize the minimum data rate or the minimum noise margin among users. The proposed loading procedures turn out to be some generalization of the waterfi lling solution used in DMT, broadened to the case when subcarriers are merged into subsets and bound with spreading sequences. Through simulations, it was shown that the energy gathering capability of the spreading function leads to a better distribution of bits and energies across the subcarriers with SS-MC-MA than with DMT. Hence, adaptive SS-MC-MA better exploits the energy resource than DMT does, so that the spared energies can be effi ciently used to increase either the throughput or the noise margin of each active user.The new adaptive system is then less sensitive than DMT to the quantifi cation loss induced by the combine limitations of DSP and fi nite order modulation constraints. Moreover, the proposed algorithms have been shown to require low computational resource. Eventually, the proposed adaptive SS-MC-MA scheme can advantageously be exploited in quasi-static multiuser environments such as PLC's, allowing an increase of either the data rate or the robustness of the systems.

Manuscript received on February 28, 2005

\section{REFERENCES}

[1] M. Crussi`ere, J. Baudais, and J. H’elard, "New loading algorithms for adaptive SS-MC-MA systems over power line channels: Comparison with DMT," in Proc. IEEE Workshop on Multi-Carrier Spread-Spectrum and Related Topics (MCSS), Sept. 2005, pp. 327-336.

[2] M. Zimmermann and K. Dostert, "A multipah model for the powerline channel," IEEE Trans. Commun., vol. 50, no. 4, pp. 553-559, Apr. 2002.

[3] _ - "Analysis and modeling of impulsive noise in broadband powerline communications," IEEE Trans. Electromagn. Compat., vol. 44, no. 1, pp. 249-258, Feb. 2002.

[4] “http://www.homeplug.org."

[5] E. Biglieri, "Coding and modulation for a horrible channel," IEEE Trans. Commun., vol. 41, no. 5, pp. 92-98, May 2003.

[6] S. Kaiser, "OFDM code-division multiplexing in fading channels," IEEE Trans. Commun., vol. 50, pp. 1266-1273, Aug. 2002.

[7] S. Mallier, F. Nouvel, J.-Y. Baudais, D. Gardan, and A. Zeddam, "Multicarrier CDMA over copper lines - Comparison of performances with the ADSL system," in Proc. IEEE International Workshop on Electronic Design, Test and Applications (DELTA), Jan. 2002, pp. 450-452. 
[8] J. Cioffi, "A multicarrier primer," ANSI T1E1.4/91-157, Committee contribution, Tech. Rep., 1991.

[9] D. Hughes-Hartogs, "Ensemble modem structure for imperfect transmission media," US patents Nos 4,679,227 (july 1987), 4,731,816 (march 1988) and 4,833,706 (may 1989).

[10] J. Campello and J. Cioffi, "Optimal discrete loading," ANSI Contribution T1E1.4/98-341, Plano, TX, November 30, 1998.

[11] S. Kaiser and W.-A. Krzymien, "Performance effects of the uplink synchronism in SS-MC-MA system," European Trans. Commun., vol. 10, July 1999.

[12] S. Hara and R. Prasad, "Overview of multicarrier CDMA," IEEE Trans. Commun., vol. 35, no. 12, pp. 126-133, Dec. 1997.
[13] A. Papoulis, Probability, random variables and stochastic processes, third edition. McGraw Hill Inc, 1991.

[14] M. Crussi 'ere, J.-Y. Baudais, and J.-F. H'elard, "Robust highbit rate communications over PLC channels: A bit-loading multi-carrier spread-spectrum solution," in Proc. IEEE International Symposium on Power-Line Communications and Its Applications (ISPLC), Apr. 2005, pp. 37-41.

[15] A. Federgruen and H. Groenevelt, "The greedy procedure for resource allocation problems: Necessary and sufficient conditions for optimality," Operations research, vol. 34, no. 6, Nov./Dec. 1986.

[16] W. Rhee and J. Cioffi, "Increase in capacity of multiuser OFDM system using dynamic subchannel allocation," in Proc. IEEE Vehicular Technology Conference (VTCSpring), vol. 2, May 2000, pp. 1085-1089. 